\title{
A theoretical approach for the interpretation of liquid metal surface tension measurements in the presence of oxygen
}

\author{
Enrica RICCI ${ }^{1)}$, Marco RATTO' ${ }^{2)}$, Elisabetta ARATO ${ }^{2)}$, Paolo COSTA ${ }^{2)}$, Alberto PASSERONE') \\ 1) Istituto di Chimica Fisica Applicata dei Materiali, CNR, Via De Marini 6 - 16149 Genova, Italy \\ 2) Dipartimento di Ingegneria Ambientale, Università di Genova, Via Montallegro 1 - 16145 Genova, Italy
}

\begin{abstract}
Theoretical models describing the transport of oxygen in metal/atmosphere systems under different fluiddynamic conditions have been developed by different authors. In the present study, as in previous ones, the molecular diffusion is the process mainly controlling the exchange of matter between the liquid metal and the atmosphere. So, in this paper a diffusional model is proposed accounting for volatile oxides and for gas phase homogeneous reactions by means of two limiting conditions: instantaneous reactions and null reactions. For the boundary conditions, the model assumes a bulk flow composition of the gas layer surrounding the liquid on the upper side and a local equilibrium constraint at the liquid interface. The asymptotic behavior of the system is described, enabling the prediction of the direction of the net oxygen flux. It has been demonstrated that the results obtained are valid for any type of homogeneous gas phase reactivity, provided that no oxide fog is formed in the gas. The model is useful to correctly guide technological processes such as single crystal growth: in the paper the application to the melt silicon/oxygen system is discussed. Finally, the present model can synthesize apparently contradictory experimental measurements of surface tension available in literature into a unique portrait.
\end{abstract}

KEY WORDS: Theoretical models, capillary phenomena, high temperature, molten silicon/oxygen, surface tension.

\section{Introduction}

Theoretical studies of oxygen transfer at the melt-gas interface have been presented in previous works, both under Knudsen [1,2] and diffusional regimes [3]. The present report presents further developments of the model under the diffusional regime [4], based on the extension of the Wagner theory [5], which regards the mechanisms of activepassive oxidation of solid metals when volatile oxides are present. Much theoretical and experimental work has been carried out on this subject for solid metals [6-9]. In contrast, significantly less effort has been applied to the examination of such mechanisms and their effect in capillary phenomena at high temperature. With the study presented here, a unifying criterion can be defined for the interpretation of molten metal surface tension measurements at different oxygen partial pressures, with a particular reference to measurements on molten $\mathrm{Si}$.

The model focuses on the diffusional gas layer surrounding the Si melt, with boundary conditions given by the bulk flow gas composition on the upper side and by the local equilibrium constraints at the liquid interface. The model is a diffusional one under stationary conditions with local equilibrium of the oxidation reactions in the gas phase, due to the high reactivity between metal vapor and oxygen. Indeed, the characteristic time of diffusion of $\mathrm{Si}$ and $\mathrm{O}_{2}$ through the layer is much smaller than the time of the oxidation reactions, implying that the chemical reaction has enough time to attain equilibrium before oxygen reaches the melt surface [10]. The assumption of local equilibrium permits the ignoring of the difficulties derived from the kinetic terms in solving the model. Furthennore it will be shown that the results presented here are valid for any type of reactivity in the diffusion layer, when no condensed oxide fog is formed. This model can be seen as an extension and generalization of the work of Wagner [5] to the case of highly reactive atmospheres.

\section{The model \\ 2.1 Assumptions}

The model refers to a fundamental geometry of an experimental apparatus for the determinations of the surface properties of metallic systems at high temperature by the sessile drop method, consisting of a tube-shaped, vacuumtight heating chamber in which a sample of a pure metal or alloy is placed and where a gaseous flux of known composition is introduced. The oxygen concentration in the gas flow can be controlled by mixing known amounts of this gas with an inert carrier, such as helium or argon, or by using gaseous mixtures like $\mathrm{CO} / \mathrm{CO}_{2}$ or $\mathrm{H}_{2} / \mathrm{H}_{2} \mathrm{O}$ able to provide oxygen as a product of a chemical equilibrium. In such mixtures the oxygen concentration is defined at each temperature by the initial concentration of the other species and by the equilibrium constant of the reaction.

With reference to this experimental layout, the model is based on the assumptions that the temperature is uniform in a large region of the tube - slaped chamber surrounding the liquid metal drop; the total pressure is constant and the gas phase is assumed to be ideal. The gas flow around the drop can be considered as laminar $\left(R e_{d}<100\right)$, while the liquid surface of the drop is considered "plane", that is, the drop diameter $d_{d}$ is "large" as far as capillary and diffusional effects are concerned. However results also hold true for the 
spherical geometry. The accumulation in the gas phase is neglected, so steady state conditions in the gaseous layer are assumed. Furthermore, the molecular diffusion is assumed to be the process that mainly controls the exchange of matter between the liquid metal and the surrounding atmosphere, i.e. a layer $\delta$ exists, surrounding the drop, in which the transport of oxygen, metal and oxide vapors is taken into account and its mechanism is exclusively diffusional. Chemical reactions in the gaseous layer are taken into account by considering two limiting kinetic conditions: the absence of reactions and the instantaneous reactions. It will be shown that under such opposite conditions, as well as for every intermediate case, the system behaves in the same way, as far as the asymptotic behavior is concerned. Hence, the results are general.

\subsection{Model equations}

Oxidation reactions with the following general stoichiometric form are considered:

$\alpha_{j} O_{2}+M=M O_{2 \alpha_{j}}$

where $\alpha_{j}$ is the stoichiometric coefficient of oxygen, $M$ is the metal and $M O_{2 \alpha}$ is the $j^{\text {th }}$ oxide, called simply $M O_{j}$ in the following.

The reaction scheme (1) has to be intended as a molecular balance and can be formally extended to any type of oxide. To do so, it is necessary to convert any stoichiometric form by assigning the value 1 to the stoichiometric coefficient of the metal, and, accordingly, all the related physico-chemical parameters such as the reaction equilibrium constants.

In the hypothesis of steady state, the local balances in the layer $0 \leq z \leq \delta$ (with the origin at the interface) can be written in terms of concentrations $c_{i}$ :

$D_{O_{2}} \frac{d^{2} c_{O_{2}}}{d z^{2}}=k \sum_{j} \alpha_{j} f_{j}$

$D_{M} \frac{d^{2} c_{M}}{d z^{2}}=k \sum_{j} f_{j}$

$D_{M O_{j}} \frac{d^{2} c_{M O_{j}}}{d z^{2}}=-k f_{j} j=1 . . n$

where $f_{j}$ is a function of the local values of $T$ and $c_{i}$, according to the Arrhenius law and the kinetic mechanism of the $j$-th reaction.

For the boundary conditions at the surface $(z=0)$ the local equilibrium is considered:

$$
\begin{aligned}
& P_{O_{2}}^{\sigma}=P_{O_{2}}^{*} ; \quad P_{M}^{\sigma}=P_{M}^{s} ; \\
& P_{M O_{j}}^{\sigma}=\min \left(P_{M O_{j}}^{s}, K_{p j} P_{O_{2}}^{*}{ }^{\alpha} P_{M}^{s}\right)
\end{aligned}
$$

On the other hand, at $\delta$ distance from the drop, a constant gas phase composition $c_{i}{ }^{0}$ is assumed, where $c_{i}{ }^{0}$ can be estimated from the material balance involving the oxygen supply, the gas outlet and the gas fluxes through the layer surrounding the metal drop[11].

In terms of dimensionless quantities, the local balances can be written as:

$$
\begin{aligned}
& \frac{d^{2} x_{O_{2}}}{d \zeta^{2}}=\Phi^{2} \sum_{j} \alpha_{j} f_{j} \\
& \frac{d^{2} x_{M}}{d \zeta^{2}}=\frac{\Phi^{2}}{\Psi_{M}} \sum_{j} f_{j} \\
& \frac{d^{2} x_{M O_{j}}}{d \zeta^{2}}=\frac{\Phi^{2}}{\Psi_{M O_{j}}} f_{j}
\end{aligned}
$$

where:

$$
\begin{aligned}
& x_{i}=\frac{P_{i}}{P_{M}}=\frac{c_{i}}{c_{M}^{s}} \quad i=O_{2}, M, 1, \ldots n ; \quad \zeta=\frac{z}{\delta} \\
& \Phi^{2}=\frac{\delta^{2} k}{D_{O_{2}} c_{M}^{s}} \Psi_{M}=\frac{D_{M}}{D_{O_{2}}} ; \quad \Psi_{M O_{j}}=-\frac{D_{M O_{j}}}{D_{O_{2}}}
\end{aligned}
$$

and the corresponding boundary conditions are:

$\zeta=0 \Rightarrow x_{i}=x_{i}^{\sigma}$ and $\zeta=1 \Rightarrow x_{i}=x_{i}^{0}$

In the limiting case of instantaneous gas-phase reactions, the following equilibrium relationships of the oxidation reactions must hold for the entire layer:

$\frac{P_{M O_{j}}}{P_{O_{2}}^{\alpha_{j}} P_{M}}=K_{p j}$

On the other hand, when no reaction takes place in the gaseous layer, all the right-hand terms of the local balances (2) and (4) are null.

\subsection{Model solution}

To solve the model, it is useful to consider the net oxygen and metal fluxes through the layer:

$$
\begin{aligned}
& N_{O_{2}}=-\left(D_{O_{2}} c_{O_{2}}^{\prime}+\sum_{j} \alpha_{j} D_{M O_{j}} c_{M O_{j}}^{\prime}\right) \\
& N_{M}=-\left(D_{M} c_{M}^{\prime}+\sum_{j} D_{M O_{j}} c_{M O_{j}}^{\prime}\right)
\end{aligned}
$$

By defining the corresponding non-dimensional quantities:

$-X_{O_{2}}^{\prime}=\frac{N_{O_{2}} \delta}{D_{O_{2}} c_{M}^{s}} ; \quad-X_{M}^{\prime}=\frac{N_{M} \delta}{D_{O_{2}} c_{M}^{s}}$

where

$$
\begin{aligned}
& X_{O_{2}}=\left(x_{O_{2}}-\sum_{j} \alpha_{j} \Psi_{M O_{j}} x_{M O_{j}}\right) \\
& X_{M}=\left(\Psi_{M} x_{M}-\sum_{j} \Psi_{M O_{j}} x_{M O_{j}}\right)
\end{aligned}
$$

the oxygen fluxes (9) can be written in the following dimensionless form:

$$
\begin{aligned}
& X_{O_{2}}^{\prime}=\left(x_{O_{2}}^{\prime}-\sum_{j} \alpha_{j} \Psi_{M O_{j}} x_{M O_{j}}^{\prime}\right) \\
& X_{M}^{\prime}=\left(x_{M}^{\prime}-\sum_{j} \Psi_{M O_{j}} x_{M O_{j}}^{\prime}\right)
\end{aligned}
$$

If the local balances are expressed in terms of the new variables $X_{M}$ and $X_{O_{2}}$, the unknown kinetic terms in the system of equations (4) disappear, giving the following two invariant relationships valid for any type of reaction regime: 
$X_{O_{2}}^{\prime}=x_{O_{2}}^{\prime}-\sum_{j} \alpha_{j} \Psi_{M O_{j}} x_{M O_{j}}^{\prime}=B_{1}$

$X_{M}^{\prime}=\Psi_{M} x_{M}^{\prime}-\sum_{j} \Psi_{M O_{j}} x_{M O_{j}}^{\prime}=B_{2}$

Hence, the net oxygen and metal fluxes for the system considered are constant and independent of their position in the layer and are also independent of the reactivity of the metal vapour, so that:

$X_{O_{2}}^{\prime}=X_{O_{2}}^{0}-X_{O_{2}}^{\sigma}=B_{1}$

$X_{M}^{\prime}=X_{M}^{0}-X_{M}^{\sigma}=B_{2}$

Hence the driving forces of the total oxygen and metal fluxes are the gradients of the variables $X_{O 2}$ and $X_{M}$, i.e. the gradient of the "Oxygen Effective Pressure":

$P_{\mathrm{O}_{2 e d}}=P_{\mathrm{O}_{2}}+\sum_{j} \alpha_{j}\left|\Psi_{\mathrm{MO}_{j}}\right| P_{M O_{j}}$

and the gradient of the "Metal Effective Pressure":

$P_{M_{e f}}=\Psi_{M} P_{M}+\sum_{j}\left|\Psi_{M O_{j}}\right| P_{M O_{j}}$

where the double contributions of the elements taken singularly and linked as oxides are clearly highlighted. Hence, the vapor pressure of oxides at the surface can be essential in determining the direction and the magnitude of the total fluxes and subsequently the average composition of the molten metal.

In fact, if $y_{\mathrm{O}_{2}}$ is the average value of the oxygen molar fraction inside the liquid metal drop, the oxygen concentration inside the liquid increases if the following conditions are attained:

$\frac{d y_{O_{2}}}{d t}>0 \Leftrightarrow X_{O_{2}}^{\prime}\left(1-y_{O_{2}}\right)-X_{M}^{\prime} y_{O_{2}}>0$

Inequalities (17) show that, even if the gas phase is under steady state conditions, this does not necessarily imply that the liquid is also under steady state conditions. Such conditions for the liquid phase correspond to the asymptotic behavior, i.e. the liquid composition $y_{\mathrm{O}_{2}}$ (and subsequently the oxygen partial pressure at the surface which must be in equilibrium with the liquid) changes until the following equality is attained between fluxes:

$X_{O_{2}}^{\prime}\left(1-y_{O_{2}}\right)-X_{M}^{\prime} y_{O_{2}}=0$.

The determination of the steady-state asymptotic conditions for the liquid metal drop is very important for the prediction of the behavior of the system. Given the relationship (18), expressed in terms of effective pressure, and given the actual initial conditions for the experimental set-up, a prediction of the oxygen flux direction for the system can be immediately made (see Fig.1)

\subsection{Asymptotic behavior}

Observing that the mean average value of oxygen solubility in liquid metals is in most cases very low (i.e. $y_{O_{2}} \approx 10^{-3}$ ), equation (18) reduces to $X_{O_{2}}^{\prime}=0$. From equations (10) and (13), the following relationship for the asymptotic behavior of a fully homogeneous layer, in terms of dimensionless concentrations, can be obtained:
$\left(x_{O_{2}}^{0}-\sum_{j} \alpha_{j} \Psi_{M O_{j}} x_{M O_{j}}^{0}\right)=\left(x_{O_{2}}^{\sigma}-\sum_{j} \alpha_{j} \Psi_{M O_{j}} x_{M O_{j}}^{\sigma}\right)(19)$

If the following boundary conditions are valid, i.e. metal is not present in the carrier gas:

$x_{M}^{0}=0 ; \quad x_{M O_{j}}^{0}=0 \quad j=1 \ldots n$,

$x_{M}^{\sigma}=x_{M}^{s} \Rightarrow x_{M O_{j}}^{\sigma}=\frac{x_{M}^{s} x_{O_{2}}^{\sigma \alpha_{j}}}{K_{j}}=g\left(x_{O_{2}}^{\sigma}\right)$

the following relationship is obtained:

$x_{O_{2}}^{0}=\left(x_{O_{2}}^{\sigma}-\sum_{j} \alpha_{j} \Psi_{M O_{j}} x_{M O_{j}}^{\sigma}\right)=$

$=\left(x_{O_{2}}^{\sigma}-\sum_{j} \alpha_{j} \Psi_{M O_{j}} \frac{x_{M}^{s} x_{O_{2}}^{\sigma_{j}}}{K_{j}}\right)$

or, equivalently, in terms of Oxygen Effective Pressure:

$P_{O_{2}}^{0}=P_{O_{2}}^{\sigma}+\sum_{j} \alpha_{j}\left|\Psi_{M O_{j}}\right| K_{P j} P_{M}^{s} P_{O_{2}}^{\sigma} \alpha_{j}$

This relationship is valid for any reaction regime where condensed oxide fogs are not present in the gaseous layer. By inverting equation (22), the asymptotic oxygen partial pressure at the liquid interface can be obtained for any given value of the partial pressure of oxygen in the feed. Moreover, remembering that $x_{\mathrm{O}_{2}}^{\sigma}$ is related to $y_{\mathrm{O}_{2}}$ by the equilibrium Sievert's law and by a mass transfer equation inside the liquid, the liquid composition can also be derived.

Furthermore, it seems important to underline that, if highly volatile oxides exist (as in the case of $\mathrm{SiO}$ ), it can happen that $x_{\mathrm{O}_{2}}^{0} \gg x_{\mathrm{O}_{2}}^{\sigma}$ at the steady state, i.e. under these conditions a nearly clean metal surface can be maintained even in the presence of a carrier gas 'rich' in oxygen.

Also the saturation conditions can be expressed as a function of temperature in terms of Oxygen Effective Pressure:

$$
\begin{aligned}
& P_{O_{2}}^{0 s}(T)=P_{O_{2}}^{s}(T)+ \\
& \quad+\sum_{j} \alpha_{j}\left|\Psi_{M O_{j}}(T)\right| K_{P j}(T) P_{M}^{s}(T) P_{O_{2}}^{s}(T)^{\alpha_{j}}
\end{aligned}
$$

where $P_{O_{2}}^{s}(T)$ is the saturation oxygen partial pressure of the first oxide condensing at the liquid surface, i. e.:

$P_{O_{2}}^{s}=\min _{j=1 . . .}\left(\frac{P_{M O_{j}}^{s}}{K_{p j} P_{M}^{s}}\right)^{\frac{1}{\alpha_{j}}}$

The curve $P_{O_{2}}^{0 s}(T)$ gives the actual values of the inlet oxygen partial pressure separating the oxidationdeoxidation conditions. If highly volatile oxides exist, the inlet saturation values $P_{O_{2}}^{o s}(T)$ will be greater than the thermodynamic values $P_{O_{2}}^{s}(T)$ by several orders of magnitude. This curve is the analogue of the liquid phase of the curve describing the transition from active to passive oxidation for solid metals [5-8]. An analogous result for molten metals has also been obtained by Laurent et al. [2] 
and Ricci et al. $[1,12]$ under the Knudsen regime. Moreover, under the deoxidation regime, the quantity $N_{M}$ gives the mole loss rate of the liquid, due to the active oxidation mechanism which allows the liquid surface to remain oxygen free.

So, with equations (22) and (23) the complete description of oxygen transport at the surface of molten metals can be made:

- equation (23) discriminates the presence of condensed oxides on the liquid surface;

- below the saturation limit (23), equation (22) discriminates the direction of the oxygen flux.

In the studies of capillary phenomena at high temperature, the conditions corresponding to equation (22) are the most relevant, equation (23) simply being the saturation limit.

\section{Application to liquid silicon}

By specifying the general definitions given in the previous section for the liquid silicon/oxygen system we have:

- oxidation reactions in the gas phase [13]:

$\mathrm{Si}+\mathrm{O}_{2} \leftrightarrow \mathrm{SiO}_{2} \quad \mathrm{~K}_{p \mathrm{SiO}_{2}}[1688 \mathrm{~K}]=9.67 \cdot 10^{15} \mathrm{~atm}^{-1}$

$\mathrm{Si}+1 / 2 \mathrm{O}_{2} \leftrightarrow \mathrm{SiO} \quad K_{p S i O}[1688 \mathrm{~K}]=7.22 \cdot 10^{13} \mathrm{~atm}^{-0.5}$

- vapor pressure of metal at $1688 \mathrm{~K}$ :

$P_{B}^{s}=P_{S i}^{s}=3.79 \cdot 10^{-7} \mathrm{~atm}$

- saturation pressures of oxygen at $1688 \mathrm{~K}$ :

$P_{\mathrm{O}_{2}}^{s}\left(\mathrm{SiO}_{2}\right)=\frac{P_{\mathrm{SiO}_{2}}^{s}}{K_{p \mathrm{SiO}_{2}} P_{S i}^{s}}=1.03 \cdot 10^{-19} \mathrm{~atm}$

$P_{O_{2}}^{s}(\mathrm{SiO})=\left(\frac{P_{S i O}^{s}}{K_{p S i O} P_{S i}^{s i}}\right)^{2}=3.69 \cdot 10^{-21} \mathrm{~atm}$

- diffusion coefficients at $1688 \mathrm{~K}$ :

$D_{\mathrm{O}_{2}}=3.61 \cdot \mathrm{cm}^{2} \mathrm{~s}^{-1}$

$D_{S i}=3.29 \cdot \mathrm{cm}^{2} \mathrm{~s}^{-1} \quad \Psi_{S i}=0.912$

$D_{\mathrm{SiO}_{2}}=2.09 \cdot \mathrm{cm}^{2} \mathrm{~s}^{-1} \quad \Psi_{\mathrm{SiO}_{2}}=-0.579$

$D_{\text {SiO }}=2.67 \cdot \mathrm{cm}^{2} \mathrm{~s}^{-1} \quad \Psi_{\text {SiO }}=-0.738$

First of all, the asymptotic behavior of the $\mathrm{Si}-\mathrm{O}$ system is considered. In Figure 1, curves representing equation (22) are plotted ( $P_{\mathrm{O}_{2}}^{\sigma}$ vs $P_{\mathrm{O}_{2}}^{0}$ ) at different temperatures. If the pair of co-ordinates representing the initial conditions of the experimental set-up is below the curve $P_{\mathrm{O}_{2}}^{\sigma}$ vs $P_{\mathrm{O}_{2}}^{0}$, a net oxygen flux towards the drop will be present and the molten metal will increase its oxygen content until the asymptotic curve is reached; if the co-ordinates of the initial conditions are above the curve, a net oxygen flux leaving the drop will exist. Curves $P_{\mathrm{O}_{2}}^{\sigma}$ vs $P_{\mathrm{O}_{2}}^{0}$ are important, since they allow the estimate of the real concentrations at the liquid surface, when the feed oxygen concentration is measured. This can also allow the interpretation, under a unifying scenario, of non-homogeneous literature surface tension measurements at different oxygen partial pressures provided by different authors, where, in some cases, the inlet $P_{\mathrm{O}_{2}}[14]$ and, in

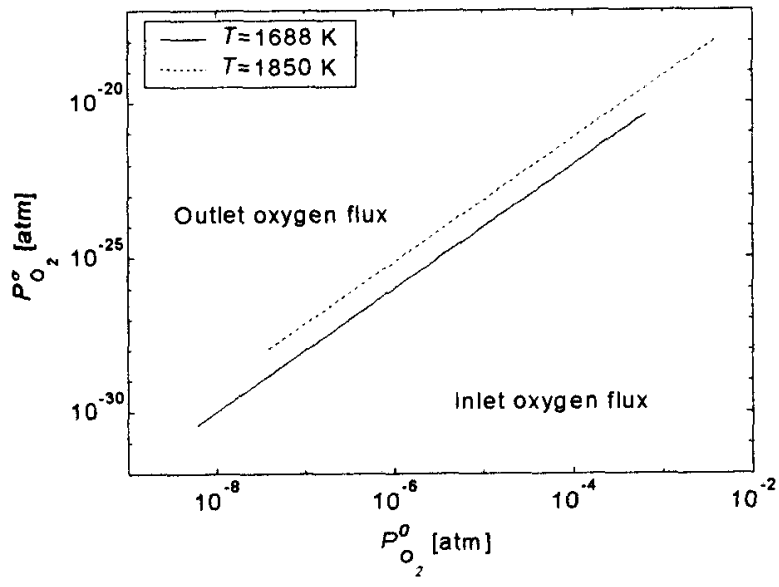

Fig. 1 Asymptotic behaviour of silicon. Curves relating interface composition to the inlet composition.

other cases, the outlet $P_{\mathrm{O}_{2}}[15]$ are measured.

As a case study, the experimental works by Huang et al [14] and Niu et al.[15] are compared. Huang et al.[15] reported surface tension measurements as a function of the oxygen partial pressure in the carrier gas fed to the furnace. Niu et al.[15], on the other hand, presented surface tension measurements as a function of the oxygen partial pressure in the exhaust gas, assumed equal to the interface partial pressure. Furthermore the two sets of experimental data were taken at very similar temperatures, near the melting point $(T=1688 \mathrm{~K}[14], T=1693 \mathrm{~K}$ [15]).

So, the experimental data by Niu et al. [15] have been kept unvaried, while the inlet oxygen partial pressure values in the axis of the abscissas of Huang et al. [14] have been transformed into partial pressure values at the liquid surface by applying equation (22).

The result of this transformation is presented in Fig. 2 The two sets of experimental results can now be plotted for similar domains of $P_{\mathrm{O}_{2}}^{\sigma}$. So, experimental results, which seemed completely contradictory, with a difference of more than 10 orders of magnitude in the oxygen partial pressure levels, belong to a unique scenario. In fact, the difference of partial pressures has been reduced to 1-2 orders of magnitude. Moreover, it seems important to underline that

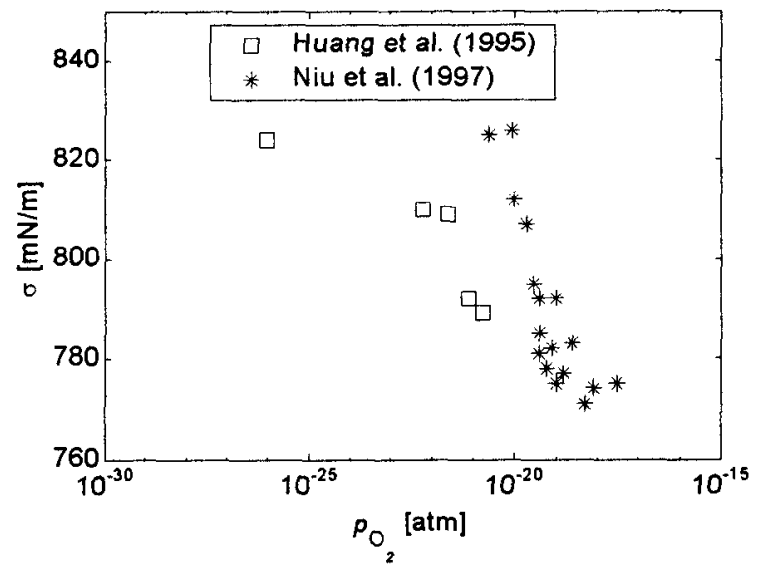

Fig. 2 Transformation of experimental data by Huang et al. by applying equation (22). 
Figure 2 has been obtained by applying literature data for the physico-chemical parameters in equation (22) and no best fit has been done. Finally, the residual difference can be significantly reduced by accounting for flow conditions in the furnace [11].

\section{Conclusions}

The results of the present research can be important for the correct interpretation of and guide to the experimental analysis and from the technological point of view. In particular they are useful for high-tech processes such as the production of silicon single crystals.

In fact, silicon single crystal growth technologies are controlled by a heat and mass transfer process, which is mainly governed by buoyancy convection and surface tension driven flow on the surface of molten metal (Marangoni effects). The phenomena of oxygen transport at the molten metal/atmosphere interface and, particularly, the mechanisms whereby the molten Si surface is kept clean, even in the presence of $\mathrm{O}_{2}$ impurities in the gas, play a very important role in this type of high tech processes.

From results obtained with this model, it is possible to explain, in accord with the Wagner theory [5], the mechanisms which keep liquid silicon oxygen-free, despite the thermodynamic driving force, due to an opposite flux of oxygen in oxide bond. Moreover, the expression of the curves of the asymptotic behavior of the system is obtained and, by means of these curves, the direction of the net oxygen flux during transients can also be predicted. So, such curves represent a fundamental tool, not only to correctly address the process of crystal growth, but for any process involving a liquid metal system under an atmosphere. In fact, equation (22) is very simple: it allows the evaluation of the composition at the liquid metal /gas interface when only the inlet composition is known and is obtained by using a model which well matches the experimental operating conditions. A comparison between different literature data of silicon surface tension measurements as a function of oxygen partial pressure is reported, demonstrating that, applying the fundamental model formula, such data can be synthesized into a unique portrait.

\section{Notation}

$c_{i}$ concentration of the $i$-th compound, $\mathrm{mol} \mathrm{cm}$

$d_{d}$ drop diameter

$D_{i}$ diffusivity, $\mathrm{cm}^{2} \mathrm{~s}^{-1}$

$f_{j}$ function (of $T$ and $c_{i}$ ) in the reaction kinetics expression of the $j$-th oxide

$k$ constant common to all reaction kinetics, $\mathrm{cm}^{3} \mathrm{~mol}^{-1} \mathrm{~s}^{-1}$.

$K_{j}$ dimensionless equilibrium constant for the $j$-th reaction, defined as $K_{j}=\left(K_{p_{j}} P_{M}^{s^{a_{j}}}\right)^{-j} \quad j=1 . . n$

$K_{p j}$ equilibrium constant for the $j$-th reaction

$M$ metal

$M O_{j} j$-th oxide

$n$ total number of oxides

$N_{i}$ molar flux, mol cm $\mathrm{cm}^{-1}$

$P_{i}$ partial pressure, atm
$R e_{d}$ Reynolds number of the drop.

$x_{i}$ concentration of the $i$-th chemical specie, normalized with respect to $c_{M}^{s}$

$X_{i}$ dimensionless oxygen/metal effective pressures

$y$ molar fraction in the liquid phase

$z$ spatial co-ordinate with origin at the liquid surface and perpendicular to it, $\mathrm{cm}$.

\section{Greek letters}

$\alpha_{j}$ oxygen stoichiometric coefficient in the $j$-th oxidation reaction

$\zeta$ dimensionless spatial co-ordinate

$\delta$ diffusive layer

$\Phi$ Thiele modulus

$\Psi$ diffusivity, normalized with respect to $D_{\mathrm{O}_{2}}$.

\section{Superscripts}

0 inlet carrier gas

$\sigma$ liquid-gas interface

$s$ saturation

- space derivative

* equilibrium with the liquid phase

\section{Subscripts}

$i$ index for all chemical species $O_{2}, M, M O_{j}$.

$j \quad$ index for oxides

\section{REFERENCES}

1) E. Ricci, A. Passerone, P. Castello and P. Costa: J. Mat. Sci., 29 (1994), 1833.

2) V. Laurent, D. Chatain, C. Chatillon and N. Eustatopoulos: Acta Met., 36 (1988), 1979.

3) P. Castello, E. Ricci, A. Passerone and P. Costa: J. Mat. Sci., 29 (1994), 6104

4) M. Ratto, E. Ricci, E. Arato and P. Costa: submitted (1999).

5) C. Wagner: J. Appl, Phys., 29 (1958), 1295.

6) C. Gelain, A. Cassuto and P. Le Goff: Oxid. Met., 3 (1971), 139.

7) E. A. Gulbransen and S. A. Jansson: Oxid. Met., 4 (1972), 181.

8) E. A. Gulbransen, K. F. Andrew and F. A. Brassart: $J$. Electrochem. Soc., 113 (1966), 834.

9) P. D. Agnello and T. O. Sedgwick: J. Electrochrm. Soc., 139 (1992), 2929

10) E. Ricci, R. Novakovic, E. Arato, M. Ratto and A. Passerone: E.L.G.R.A. News, 21 (1999), 117.

11) M. Ratto, E Ricci and E Arato: in preparation.

12) E.Ricci, L. Nanni, E.Arato and P. Costa: J.Mat. Sci., 33 (1998), 305.

13) O. Knacke, O. Kubashewski and K. Hesselmann: Thermochemical properties of inorganic substances, II ed. (1991) Springer Verlag, Düsseldorf.

14) X. Huang, S. Togawa, S.-I. Chung, K. Terashima and S. Kimura: J. Crystal Growth, 156 (1992), 52.

15) Z.Niu, K.Mukai, Y.Shiraishi, T.Hibiya, K.Kakimoto and M.Koyama: Proc. Int. Conf. "High Temperature Capillarity", Ed. by N.Eustathopoulos and N.Sobczak, Krakow, Poland, (1997), 175. 